\title{
VERS UNE APPRÉHENSION FINE ET CRITIQUE DU DISCOURS ARGUMENTATIF AU NIVEAU C1/C2 DU CECR
}

\author{
Enseigner les compétences interprétatives à l'oral. Cadre rhétorique et \\ générique du débat, par Bernadeta Wojciechowska, \\ Wydawnictwo Naukowe UAM, Poznań 2017, 230 pp., \\ ISBN: 978-83-232-3207-0. \\ https://doi.org/10.19195/0557-2665.67.17
}

Le domaine de référence de la monographie de Bernadeta Wojciechowska est la didactique des langues étrangères aux niveaux $\mathrm{C} 1 / \mathrm{C} 2 \mathrm{du} \mathrm{CECR}^{1}$ dans le cadre de l'activité de réception orale. Le choix des niveaux avancés mérite une attention du fait qu'il s'inscrit dans un terrain relativement peu fréquenté par les didacticiens des langues étrangères (désormais : LE), et plus précisément du français langue étrangère (FLE), du fait de la rareté des groupes d'apprenants. Néanmoins, quelque peu nombreux qu'ils soient, les étudiants des philologies françaises, les élèves des classes bilingues et les (futurs) professionnels des lettres ayant acquis un haut degré de compétence en français, demandent une didactique sans cesse renouvelée et faisant face aux défis d'un monde en constante mutation. L'une des compétences hautement requises à un stade avancé d'apprentissage, auprès d'un public (potentiellement) élitiste, est « l'appréhension fine et critique du discours argumentatif » ${ }^{2}$, dont le discours médiatique, devenu un vrai dédale de sens/contre-sens/faux sens, espèce de sable mouvant pour le public d'aujourd'hui, ballotté en permanence entre information et désinformation. L'appropriation des stratégies d'écoute dépassant le cadre habituel de la reconstruction des (sens des) énoncés est une véritable obligation pour les citoyens des pays démocratiques, car leur maitrise est en train de devenir un instrument de pouvoir social. En cernant l'interprétation de sens en tant que compétence plurielle, hétérogène, tributaire du contexte social de sa production et notamment du genre du discours, l'objectif de l'ouvrage est de fournir, comme l'indique le titre, un cadre rhétorique et générique, susceptible d'être réinvesti dans la dimension pédagogique, des compétences interprétatives des débats radiodiffusés ${ }^{3}$.

${ }^{1}$ Le Conseil de l'Europe, Le Cadre Européen de Référence pour les Langues, Didier, Strasbourg 2001 (<https://rm.coe.int/16802fc3a8 $>$ ).

2 Bernadeta Wojciechowska (p. 9) proclame l'acquisition des compétences interprétatives : « l'objectif suprême, l'aboutissement escompté de toute éducation et le gage de l'autonomie intellectuelle de l'apprenant».

${ }^{3}$ Force est de constater que le discours argumentatif est présenté lui-même de façon très stéréotypée dans les méthodes de langues étrangères et presque exclusivement dans le cadre des activités de production, à travers la rédaction des arguments et l'étude des connecteurs logiques. 
Le livre est divisé en cinq chapitres suivis d'un bilan et des perspectives des recherches. Le premier chapitre est consacré à l'interprétation du sens - une forme avancée de la compréhension orale. L'auteure souligne le besoin de conceptualisation didactique de cette compétence, laissée pour compte par la didactique de l'oral, limitée souvent à des activités de repérage et à la compréhension « sur le mode automatique » (p. 16), distincte de la compréhension « sur le mode interprétatif» (ibidem). Celle-ci doit être envisagée par rapport aux processus cognitifs, premièrement, dans le cadre des modèles de l'esprit humain, dont l'une des propriétés exclusives (prises en charge par les modèles culturalistes, mais pas par les modèles computationnels) est d'inférer une intention de communication; deuxièmement, dans celui de la théorie des schémas conceptuels, susceptibles entre autres de rendre compte du phénomène de la mémoire collective élaborée et véhiculée, par exemple, par les médias ; finalement, dans celui des modèles de compréhension orale en LE (qui n'expliquent pas forcément comment s'articulent les processus ascendants et descendants de construction du sens lors de la compréhension orale interprétative...). Celle-ci est par ailleurs absente des objectifs du CECR, ce qui conforte les postures d'écoute limitées à la restitution du contenu et n'est pas favorable à l'élaboration du sens, laquelle est davantage liée à l'analyse critique de segments lexicaux dans leur fonction discursive. En reconnaissant le besoin d'automatisation de la compréhension en LE en tant qu'objectif didactique aux niveaux A1-B2, Wojciechowska insiste sur les dangers d'adoption d'une telle approche comme seule valable jusqu'aux niveaux $\mathrm{C} 1-\mathrm{C} 2$. Ces dangers sont le verbocentrisme, la littéralité et le dogmatisme socio-culturel. À l'opposé, l'interprétation du sens, activité métadiscursive, critique et réflexive, permet de découvrir le sens profond d'un événement discursif, en l'ancrant dans des contextes plus larges que l'immédiat et en le confrontant à la logique de son domaine de référence. Le chapitre se termine sur une esquisse d'inventaire des compétences interprétatives de l'apprenant saisies en termes de savoirs, savoir-faire et savoir-être, suivi d'un classement des conditions favorables à la formation de ces compétences, à savoir : le ralentissement de l'écoute (voire le recours aux transcriptions), la gestion métadiscursive et métacognitive de la tâche par l'apprenant lui-même, finalement, le guidage didactique réfléchi et progressif des compétences interprétatives dans le processus d'enseignement.

Le deuxième chapitre a le mérite de se focaliser sur les théories de l'argumentation susceptibles d'être appliquées avec succès sur le terrain de la didactique de l'interprétation du discours médiatique. Wojciechowska n'hésite pas à opter pour la théorie aristotélicienne, non seulement à cause de son réinvestissement constant dans les sciences du langage, mais surtout pour son opérationnalité dans l'interprétation des données socialement indexées dans le discours. La première méthode d'argumentation, relevant du premier élément de la triade classique - le logos - , se manifesterait notamment à travers le raisonnement démonstratif, déductif par excellence et attaché à la logique formelle, donc d'une utilité limitée pour les objectifs interprétatifs dans l'apprentissage des LE. La deuxième méthode - la dialectique - a une visée spéculative et « cherche à développer des méthodes de résolution de problèmes idéologiques [...] face à une divergence des points de vue » (p. 63). Elle semble plus propice à une implantation didactique, à cette réserve près que les dialogues purement heuristiques sont rarissimes dans l'espace social (d'habitude, les instances énonciatives sont fonctionnelles, comme par exemple un avocat, qui représente une position prédéterminée et ne recherche pas la vérité). Finalement, la rhétorique, qui a une visée persuasive et qui s'applique à la détermination du juste, du bon et du 
beau, est de loin la plus intéressante du point de vue des objectifs de l'étude du fait de son enracinement dans les pratiques discursives d'une communauté, intégrant «la crédibilité et la légitimité socio-discursive du locuteur (ethos) et son adaptabilité aux valeurs et sentiments de l'auditoire (pathos) » (p. 68). Dans le contexte exolingue, elle permet notamment la décentralisation de l'apprenant par rapport à ses propres valeurs (ce qui est une des idées phares de l'enseignement/apprentissage interculturel à l'époque du CECR).

Dans la deuxième partie du chapitre, l'auteure redéfinit plus précisément les compétences interprétatives de l'apprenant à la lumière de la rhétorique, en mettant en exergue leur caractère fonctionnel et interactif et la place qu'y occupent les catégories aristotéliciennes du pathos (par exemple l'expression des émotions mais aussi la recherche de la connivence), de l'ethos (par exemple la représentation sociale de l'énonciateur et le reflet des valeurs morales de la communauté) et du logos (le raisonnement logique de déduction et le raisonnement dialectique d'induction, source d'implicites) — qui demandent absolument une didactisation explicite dans le cadre d'activités d'écoute des discours argumentatifs.

Finalement, après avoir esquissé une typologie de techniques argumentatives, l'auteure entame la discussion sur une question de taille concernant le choix de l'approche, descriptive ou normative, dans la modalisation de l'enseignement des compétences interprétatives.

Le troisième chapitre est orienté plus concrètement vers les questions du guidage de l'interprétation de l'argumentation à travers le genre discursif, dans une approche intégrant les enjeux de l'argumentation. Wojciechowska puise avec brio dans les apports de plusieurs courants linguistiques : analyse du discours, pragmatique anglo-saxonne, analyse des interactions, analyse conversationnelle. Cela étant, la catégorie de genre, discutée en profondeur en tant que catégorie socio-cognitive, un objet de conscience et un cadre interprétatif quotidien, est pour elle cruciale puisque, constituant un palier entre le texte et le discours, elle permet de fédérer les outils rhétoriques susceptibles d'une didactisation pour des objectifs interprétatifs. Le chapitre a le mérite d'opérer un choix épistémologique concernant le placement du discours argumentatif parmi les concepts de genre, de discours et de texte, en légitimant par conséquent l'activité de l'interprétation dans le cadre du texte compris comme unité du discours, « qui se concrétise toujours dans un espace social » (p. 105). Cette décision est lourde de conséquences, puisqu'elle motive une approche par cadre générique dans l'enseignement des compétences interprétatives, dont le potentiel est illustré au point 3.3.1. (pp. 118-120) à l'aide de la métaphore scénique (scène englobante, scène générique, scénographie) empruntée à Dominique Maingueneau. Cependant, elle est motivée a fortiori par le phénomène fréquent de l'hybridation des genres - résultant justement de la compétence ou de la sensibilité générique des membres d'une communauté. Dans une approche interculturelle, l'interprétation des genres dans le cadre de différents groupements (par ex. familles de genres, hypergenres, « chaînes » de genres...) peut être source d'une réflexion très enrichissante aux niveaux supérieurs du CECR, en permettant notamment d'opérer le tri entre les réalisations des pratiques discursives régulières (sociales) et accidentelles (individuelles). À cela s'ajoute «l'intensité » (p. 127) interactive du discours argumentatif qui se joue à plusieurs niveaux et qui peut devenir un terrain de prédilection pour l'étude réfléchie des stratégies rhétoriques des interactants. D'autres pistes d'exploration de l'enseignement des compétences interprétatives du discours argumentatif passent par l'analyse des actes de parole et par le contrat de communication dans 
le cadre duquel il se produit, ainsi que par la modularité du genre et l'établissement des séquences contribuant à son « déroulement» (p. 131).

Dans le quatrième chapitre, l'auteure modélise un débat médiatique en LE sur l'exemple de Du grain à moudre, émission de France Culture, en commençant par la définition de l'hypergenre débat, qui a pour propriétés définitoires le fait d'être public, non-conclusif, normatif, polylogal et formalisé ; sa finalité dominante est rhétorique. Le genre débat, à son tour, se caractérise notamment par son inscription dans le discours médiatique (sa scène englobante) avec certaines conséquences pour son exploitation didactique. Par ailleurs, le discours médiatique réalise simultanément deux logiques : démocratique et commerciale (un spectacle à tonalité polémique accrue), ce qui peut être source de tensions dont les enjeux médiatiques sont discutés par Wojciechowska en termes rhétoriques de docere, movere et delectare, susceptibles de guider les stratégies interprétatives des apprenants, qui, force est de le constater, le relient d'habitude au genre informatif, et pas au persuasif. Le chapitre définit finalement les enjeux interprétatifs du genre visé par son ouvrage, à savoir du débat radiodiffusé, avec un accent mis sur la nécessité de la prise en compte des paramètres extralinguistiques véhiculés par les voix dans leur fonction rhétorique (et pas seulement comme élément constitutif de l'ethos). Parmi les sous-catégories du débat, Wojciechowska choisit le débat-citoyen (plutôt que le débat-spectacle ou le débat-témoignage) comme la meilleure référence pour l'apprentissage des stratégies interprétatives - non sans envisager sa mission démocratique (explicite dans le cas de l'émission étudiée), paradoxalement moins réalisée à travers la reconnaissance des opinions que par le décryptage de schémas interprétatifs. L'analyse du plan interactif (préconstruit par des instances médiatiques dans le cas analysé) permet de mettre en relief la relation entre les enjeux d'information et de captation dans un tel débat, en se penchant sur le rôle du journaliste et les difficultés qui peuvent affecter la formulation des «hypothèses interprétatives » (p. 158) par les apprenants compte tenu de la dynamicité de l'interaction et des recadrages participatifs. Une des questions soulevées concerne la posture d'écoute de l'apprenant en tant que destinataire tertiaire (le premier étant présent dans le studio, le deuxième étant le public étranger) - face au destinataire (secondaire) de l'émission. Wojciechowska recommande comme un des axes d'interprétation du débat le ciblage du destinataire et le positionnement socio-discursif par rapport à celui-ci. Néanmoins, le travail interprétatif peut, voire doit être facilité par le découpage du débat en séquences rhétorico-discursives de moindres dimensions (vu déjà la charge mnémotechnique en LE) pour, obligatoirement, dépasser ce morcellement provisoire dans l'étape cruciale de réflexion sur la négociation du logos, de l'ethos et du pathos par les participants de l'émission. L'auteure cerne avec précision les objectifs pédagogiques possibles à atteindre lors d'un travail interprétatif autour des trois étapes principales d'un débat, qui sont : le bloc introductif, à caractère monologal (titre, chapeau, présentation des invités), le premier tour de table (étape transitoire lors de laquelle l'ethos des participants est renégocié) et le débat proprement dit qui, à travers des « séquences rhétoriques » (cf. p. 172), ainsi que les types de progression : heuristique et contre-argumentative (p. 174), doit permettre d'interpréter le sens de l'émission d'un côté, et l'apport de chacun des participants de l'autre.

Le modèle didactique de guidage interprétatif respectant la fonctionnalité rhétorique de chaque étape peut donner lieu à plusieurs séquences didactiques ayant pour but, en premier lieu, la conscientisation « quant à la manière dont le type de discours médiatique et le genre du débat déterminent le type de raisonnement et d'argumentation déployés par les 
différentes instances construisant le débat » (p. 184). Cette conscientisation s'effectue grâce à la réalisation de quelques principes de base qui sont : la conceptualisation des objectifs de l'écoute interprétative par l'apprenant même, la mise en place d'un métalangage adéquat et univoque, et le développement des connaissances rhétoriques en relation avec les catégories de logos, ethos et pathos dans le cadre d'un entraînement stratégique. Une expérience-pilote effectuée par la chercheuse corrobore la validité du modèle intégrant la distinction des séquences relativement stables à leur dynamique rhétorique interne et à la transversalité des stratégies rhétoriques, en munissant l'apprenant des outils permettant une supervision métacognitive de leur activité d'interprétation dans le cadre d'un entraînement stratégique.

Tout porte à croire qu'une telle approche des compétences interprétatives est susceptible de fournir un apport majeur au développement des compétences métacognitive et métadiscursive des apprenants. En effet, la monographie a le mérite d'ouvrir le débat sur les compétences interprétatives en LE, considérées par le CECR comme un axiome et non définies. Il ne nous reste qu'à espérer la publication de résultats confirmant la validité du modèle proposé dans d'autres contextes, d'autant plus qu'il n'est pas exclu que les problèmes indiqués dans l'ouvrage concernent au même titre l'interprétation des discours médiatiques en langue maternelle.

Monika Grabowska ORCID: 0000-0001-7828-0821

Université de Wrocław monika.grabowska@uwr.edu.pl

\section{L'INCONTOURNABLE EMPRUNT}

L'emprunt en question(s). Conceptions, réceptions, traitements lexicographiques, sous la direction d'Alicja Kacprzak, Radka Mudrochová et Jean-François Sablayrolles, Éditions Lambert-Lucas, coll. « La Lexicothèque », Limoges 2019, 200 pp., € 24 (paperback), ISBN: 978-2-35935-230-6. https://doi.org/10.19195/0557-2665.67.18

En 2013, en rendant compte d'un ouvrage entièrement consacré au traitement lexicographique de l'emprunt, nous avions souligné que ce phénomène linguistique restait alors au centre de la préoccupation des spécialistes ${ }^{1}$. Une demi-douzaine d'années plus tard, cette constatation n'a rien perdu de son actualité. En témoigne, entre autres, l'activité des Éditions Lambert-Lucas, qui, dans leur collection «La Lexicothèque », dirigée par Christine Jacquet-Pfau et focalisée sur des questions relatives aux unités lexicales

${ }^{1}$ Voir W. Ucherek, compte-rendu de Les dictionnaires et l'emprunt. XVI $-X X I^{e}$ siècle, A. Steuckardt, O. Leclercq, A. Niklas-Salminen et M. Thorel (dir.), Publications de l'Université de Provence, Aix-en-Provence 2011 (Romanica Wratislaviensia LX, 2013, pp. 173-175). 